\title{
Adequate Reverberation Time for Brass Band Auditoriums
}

\author{
Ignacio Guillen, Ana Llopis, Antonio Uris \\ Departamento de Física Aplicada, Escuela Técnica Superior de Arquitectura, \\ Universidad Politécnica de Valencia. Camino de Vera, 14, 46022 Valencia, Spain
}

(Received 20 November 2000; revised 20 October 2001; accepted 17 December 2001)

\begin{abstract}
Music bands are deeply rooted in the Valencian Community. According to the particular acoustical characteristics of brass bands, some special requirements have to be considered regarding their indoor performances. This paper studies two different auditoriums designed for brass band performances. 'Palau de la Música' Auditorium (Bunyol. Valencia) was built in 1950 and had a reverberation time of around $0.80 \mathrm{~s}$ at $1000 \mathrm{~Hz}$. The original acoustical conditions gave good overall results and accordingly, they were maintained after the Auditorium's reconstruction in 1995-1997. 'Quart de Poblet Moli Vell' Auditorium (Quart de Poblet. Valencia) was built in 1994-1998, to be used both for brass band concerts and theatrical performances. Its acoustic design was carried out following the 'Palau de la Música' Auditorium, whose performance results were considered satisfactory.
\end{abstract}

\section{INTRODUCTION}

A brass band can be defined as an instrumental group in which brass and percussion sections preponderantly take part. A regular brass band distribution pattern consists of 8090 per cent brass, 10-20 per cent percussion and on occasion a small percentage of string instruments.

Music bands are an important sociological phenomenon in the Valencian Community, with bands existing in almost every town. Music bands and the musical associations designed to protect them act as real meeting points, focusing their activity on cultural dissemination though concerts, conferences, courses and festivals, as well as on training the band's future instrumentalists.

From the beginning, music bands have traditionally performed in open spaces, as they walked along the streets or in festivals and public acts hosted at open-air auditoriums. The tendency towards indoor performance is quite recent, and new acoustical requirements have to be considered for auditoriums hosting this kind of event.

There are usually around 130 instrumentalists playing in each concert, but it is not unusual to find more than $150 \mathrm{mu}-$ sicians on stage. ${ }^{1}$

The dynamic range of brass instruments was measured by Miskiewicz and Rakowski ${ }^{2}$ in terms of sound-pressure level (SPL) or loudness level (LL) showing that the dynamic LL range could exceed the SPL one, depending on the broadening degree of the spectral envelope with playing levels. This increase becomes greater in brass instruments with a difference of about $6 \mathrm{~dB}$. The LL dynamic range varies with each instrument played and each scale segment performed, from around $15 \mathrm{~dB}$ to $30 \mathrm{~dB}$, being greater in high frequencies. From a "pianissimo" with a sound pressure level of about $70 \mathrm{~dB}$, to a "fortissimo" with a level about $105 \mathrm{~dB}$, one can see the hall should be able to provide adequate perception of the music everywhere.

Both the instantaneous sound pressure level and the equivalent continuous sound level $\left(L_{e q}\right)$ for the measurement period, depend on the instrument being played and the music performed, as well as on the position of the musician on stage. Royster et al. ${ }^{3}$ have measured the equivalent daily 8-hours exposure sound pressure level $\left(L_{e q}\right)$ of a typical brass and percussion ensemble during rehearsal time. The levels measured ranged between $L_{e q}^{\min }=79 \mathrm{dBA}$ and $L_{e q}^{\max }=99 \mathrm{dBA}$, with a mean value of $L_{e q}^{\text {mean }}=89.9 \mathrm{dBA}$.

This energy cannot be radiated evenly in all directions due to the acoustical shadow produced by the instruments and the musicians alike. Although each instrument has a particular directional diagram, usually wind instruments radiate sound omnidirectionally at low and medium frequencies $(500-650 \mathrm{~Hz})^{4,5}$ creating a spherical field. With increasing frequency, the emission angle decreases and approaches the instrument's axis.

Considering the number of musicians involved in each concert and the particular distribution of brass and percussion sections, the high sound directivity and acoustic power that the band can reach in a determinate interpretative moment can be easily inferred.

The music performed by bands has a strong component of low frequencies and a highly directional sound projection, reaching extremely high sound pressure levels during concerts hosted in auditoriums. These particularities ask for special acoustical requirements regarding auditorium performances.

In those auditoriums in which musical events share their space with theatrical performances, there are two clearly differentiated spaces: The stage tower and the seating area.

Synchronisation among musicians in a brass band is based on the ability to detect accurately fellow musician's performance timing. ${ }^{6}$ Ceiling reflections on the musicians' platform are therefore needed in order to facilitate the musicians' need to listen to each other. An acoustical shell is desirable.

Sound produced in small auditoriums can reach very high levels of reflected sound energy if not enough absorption is present. To avoid this situation sound absorbing materials can be introduced but that leads inevitably to an excessive decrease of the reverberation time.

This great intensity of sound together with the small volume this type of auditorium require the moderation of rever- 